\title{
Factors affecting perceived orientation of the Poggendorff transversal*
}

\author{
DAVID H. KRANTZ $\dagger$ and DANIEL J. WEINTRAUB \\ University of Michigan, Ann Arbor, Michigan 48104
}

\begin{abstract}
The Poggendorff figure was simplified by removing the right transversal segment. When Ss judged the distance between a dot located on the right parallel and the imagined point where the left transversal, if extended, would intersect the right parallel, the error was independent of dot location. This result is consistent with the idea tnat the Poggendorff figure is processed asymmetrically by (mis)projecting one of the transversals across to the opposite parallel. Systematically omitting line segments reduced (and sometimes reversed) illusory effects. The most critical Poggendorff feature was the obtuse angle formed between transversal and parallel. Ss vertically adjusted one of two dots to apparent collinearity with an implied transversal, the tip of an intervening vertical line and the other (stationary) dot. Which dot was stationary proved critical. This primitive Poggendorff display generated no illusion unless the implied transversal, defined by the stationary dot, was the one that formed an obtuse angle with the vertical line. This result also strongly supports asymmetric active processing ideas. Perceived orientation is a property of directed line segments.
\end{abstract}

The standard Poggendorff figure is composed of two parallel lines cut by a transversal that is interrupted between the parallels. The illusion consists in the apparent noncollinearity of the transversal segments; e.g., in Fig. 1, the right-hand transversal segment looks as though it should be translated downward in order to be collinear with the left-hand segment.

Weintraub and Krantz (1971) showed that the effect of stimulus variables on illusion magnitude is conveniently described if we consider that the perceived orientation of each physically present transversal segment, relative to the imagined central transversal segment, is distorted by the parallel lines. The most critical element in producing such distortion is the segment of a parallel that makes an obtuse angle with an outer transversal segment, i.e., the upper left parallel segment or the lower right one. For example, if the whole right half of the figure is deleted, replacing the intersection of the right-hand parallel and right-hand transversal segment by a point, a very large illusion is still obtained; whereas, if one merely reduces the contrast of the segment of the parallel that forms the obtuse angle, one produces a regular reduction in illusion, approaching zero illusion for zero contrast.

Weintraub and Krantz concluded that it is unreasonable to aim for a unitary explanation of the Poggendorff illusion. What is needed, rather, is a general theory of the various factors that affect perceived orientation of line segments. The theory would then be applied to many kinds of figures, including the Poggendorff.

This paper reports some additional experiments, in

*We thank A nita Caplan, Robert Grimshaw, and Lillian Tong for their assistance and suggestions. Support was provided by NSF Grant GB 8181 and by a USPHS Research Scientist Development Award (K3-MH-35, 253) to the second author.

†Address: Human Performance Center, University of Michigan, Perry Building, 330 Packard Road, Ann Arbor, Michigan 48104. which we measured illusion magnitudes produced by perturbations of the Poggendorff figure. The results bear on the construction of theories of perceived orientation. For convenience of cross reference, we report these results as Experiments VI, VII, VIII, and IX, continuing the numbering of Weintraub and Krantz (1971).

\section{EXPERIMENT VI}

\section{Method}

Figure 1A depicts one of the 10 stimuli used in this experiment, with the addition of calibration lines showing the definition of the independent variable, $x$, which varied across these stimuli. The $S$ was initially shown a sample figure, which was like 1A except that the transversal continued to the right parallel, so that the true intersection of the transversal and parallel was represented. He was told that in test figures, he should imagine where the intersection would be if the transversal line were extended, and was required to judge the position of that imagined intersection relative to the position of the black dot (a minor paraphrase of the instructions to Ss). The judgment was made using the configuration depicted in Fig. 1B. A movable open circle represented the imagined intersection; the $S$ slid this circle along the line until its position, relative to the black dot in Fig. 1B, matched the position of the imagined intersection relative to the black dot in Fig. $1 \mathrm{~A}$.

The independent variable was the signed distance, $x$, between the true intersection and the center of the black dot in Fig. 1A; the dependent variable was the signed distance, $y$ (center-to-center), between the open circle and the black dot in Fig. 1B; the illusion was measured by $y-x$. The coordinates, $x$ or $\mathrm{y}$, are taken to be positive if the black dot is above the true intersection (Fig, 1A) or the open circle (Fig. 1B); in the example shown, they are positive.

The values of $\mathrm{x}$ ranged from -35 to $+10 \mathrm{~mm}$, in steps of $5 \mathrm{~mm}$.

The differences between this method and the ones we have used in previous studies will be discussed below.

Stimuli were drawn in India ink on white paper. Lines were $0.2 \mathrm{~mm}$ thick; the diameter of the black dot was $1.5 \mathrm{~mm}$. The two parallel lines, $22 \mathrm{~cm}$ in length, were centered on the $8 \frac{1}{1 / 2} \mathrm{x}$ 11 in. $(21.6 \times 27.9 \mathrm{~cm})$ sheet, and were separated by $29 \mathrm{~mm}$ (center to center). The transversal segment. $9 \mathrm{~cm}$ in length. intersected the left parallel at an angle of $24.2 \mathrm{deg}\left(\tan ^{-1} 0.45\right)$. $12 \mathrm{~cm}$ above the bottom of the sheet. 


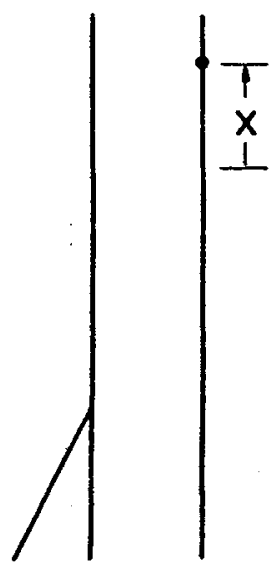

(A)

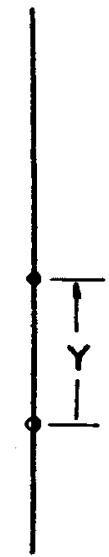

(B)
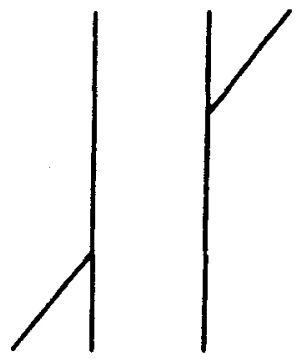

\section{STANDARD} FIGURE

Fig. 1. Lower right: The standard Poggendorff display. The transversal segments are collinear but appear to be translated apart vertically. The displays studied were all modifications of this standard one. (A) Display that was judged in Experiment VI. The right-hand transversal was replaced by a black dot on the right-hand parallel. Distance $x$ is measured from the true intersection of the continued transversal with the right-hand parallel to the center of the black dot; $x$ is positive when the dot is above the true intersection, as it is in the example shown. (B) Measuring apparatus for Experiment VI. The open circle was moved vertically until its direction and distance from the black dot matched the direction and vertical distance (in Display 1A) of the imagined intersection from the black dot. Coordinate $y$ is positive when the dot is above the open circle, as it is in the example shown. The illusion measure was $I=y-x$. (A and $B$ : Construction lines showing $x$ and $y$ were not present in the displays shown to the Ss.)

The measuring apparatus (Fig. 1B) consisted of a black line running down the center, the full length of a $9 \times 12$ in. $(22.9 \times$ $30.5 \mathrm{~cm}$ ) sheet, with a black dot at its midpoint. The widths of the line and dot were 0.2 and $1.5 \mathrm{~mm}$, respectively. The open circle, also $1.5 \mathrm{~mm}$ in diam, was drawn on the underside of a clear acetate overlay. When the $S$ slid the acetate overlay, with its left edge flush against a raised board, the open circle remained centered on the line.

The stimulus sheets were placed to the left of the measuring apparatus separated by $55 \mathrm{~mm}$ at the bottom and by $67 \mathrm{~mm}$ at the top, thus forming an angle of $2 \frac{1 / 2}{\mathrm{deg}}$. The $S$ turned slightiy left to view the stimulus, slightly right to view the measuring apparatus; the parallels and the measuring line were therefore aligned with the midplane of his visual field. Viewing distance was approximately $60 \mathrm{~cm}$, varying somewhat from $S$ to $S$.
The $50 \mathrm{Ss}$ were undergraduates at The University of Michigan, paid for participation. They had (corrected) acuity in the normal range and had no previous experience in visual-illusion experiments. Each $\mathrm{S}$ was instructed as outlined above, and then he produced settings for all 10 stimulus sheets $(x=-35 \mathrm{~mm}$ to $\mathrm{x}$ $=+10 \mathrm{~mm}$ ) in a random order. He then made a second set of settings, in a different random order. (Randomization was different for each S.) Following each of these 20 settings, the $E$ measured the coordinate, $y$, in full view of the $S$. (Note that the $S$ did not obtain any information about the true value of the distance, $x$, in this way.)

\section{Results}

The mean values of $y$, across the $50 \mathrm{Ss}$, are shown in Fig. 2, as a function of the true coordinate value, $x$, and of first or second attempt. The standard error of the mean is shown for each point by an error bar in one direction. The dashed line shows the relation expected if the judgments are veridical. The settings from first judgments are extremely well fit by a constant illusion size, $y-x=13.5 \mathrm{~mm}$. The second judgments indicate a slightly decreasing illusion, from over $15 \mathrm{~mm}$ at $\mathrm{x}=-35 \mathrm{~mm}$ to around $12 \mathrm{~mm}$ at $\mathrm{x}=+10 \mathrm{~mm}$. We are uncertain whether this interaction is replicable.

\section{Discussion}

The stimuli used in this study (Fig. 1A) modify the standard Poggendorff figure by substituting a black dot for the right-hand transversal segment. Weintraub and

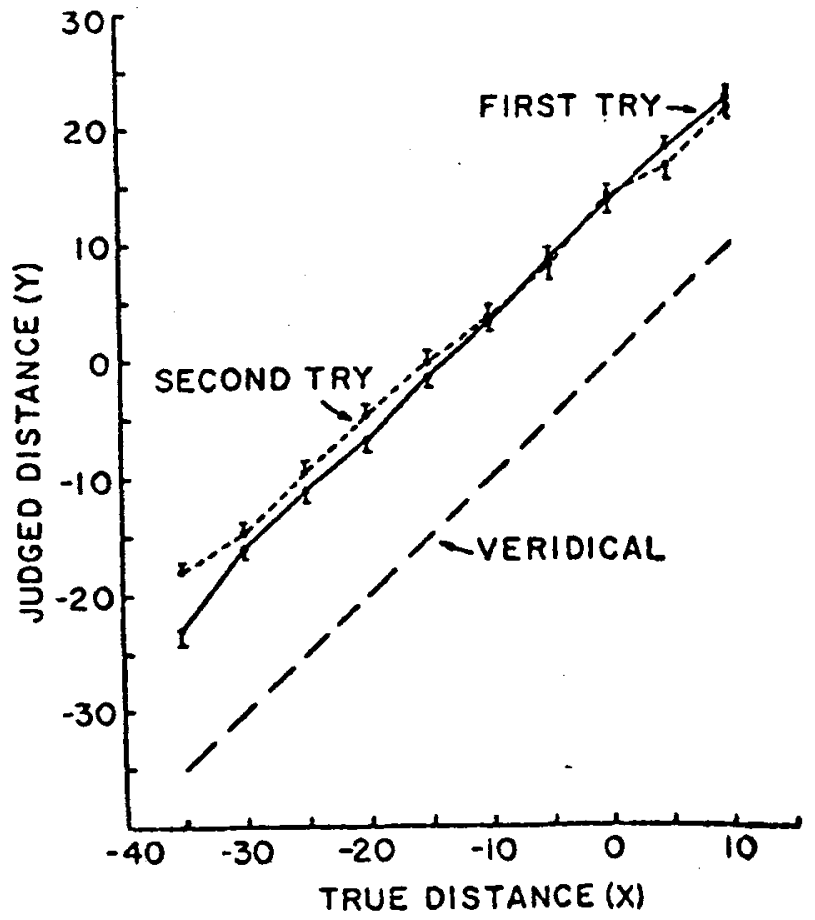

Fig. 2. Mean judged distance ( $y$ in Fig. 1B) as a function of true distance ( $x$ in Fig. 1A) from Experiment VI. First-series and second-series judgments (obtained from each $S$ ) are plotted separately. Error bars show standard errors of the means, based on variability among the 50 Ss. 
Krantz (1971) showed that this substitution leaves the illusion almost unchanged (perhaps slightly enhanced). The method used there consisted of moving the black dot until it appeared to lie on the continuation of the left transversal (either method of adjustment or up-and-down method). Thus, the illusion was always measured by finding the position of apparent collinearity. The present method permits measurements outside this position.

The results indicate that the inferred location of the perceived intersection of the transversal with the right-hand parallel is independent of the position of the dot on the right-hand parallel. Such an invariance result suggests (though it does not prove) that this perceived intersection is an essential element in the Poggendorff illusion. Both Experiment II of Weintraub and Krantz (1971) and this result indicate that the symmetry of the classical Poggendorff figure is not of basic importance. The results are what one would expect if the figure were processed asymmetrically, by projecting the left transversal segment across to the right-hand parallel or by projecting the right transversal segment back to the left parallel, or both.

\section{EXPERIMENT VII}

\section{Stimulus Configurations}

The simplified Poggendorff figure has four line segments (labeled in Stimulus 1 of Fig. 3): Segment a, the transversal; Segment $b$, the part of the left parallel forming an obtuse angle with a; Segment $c$, the acute-angle part of the left parallel; and Segment $d$, the right-hand parallel (which we do not subdivide into the parts above and below the black dot). Previously (Weintraub \& Krantz, 1971, Experiments III and IV), we showed that reducing the contrast of $b$ reduces the illusion, with little, if any, illusion when $b$ is omitted, whereas omitting $a, c$, or $d$ produces only a moderate reduction in the size of the illusion.

In order to obtain a clearer idea of the contributions of different factors to the illusion, we studied the 16 stimulus configurations consisting of all possible subsets of Segments a, b, c, d, including both the empty subset, where there are only three dots and the $S$ must decide whether the right-hand one is collinear with the first two, and the full set, which coincides with the configuration used in the previous experiment. These 16 stimuli and their designations in terms of $a, b, c, d$ are shown in the left columns of Fig. 3. Note that when a is missing, its direction is defined either by two black dots (Configurations $4,8,12$, and 16 ) or by one black dot and the tip of Segment $b$ or Segment $c$ (Configurations $10,11,13$, and 14). The leftmost dot lay along the path of the missing transversal segment, a. $60 \mathrm{~mm}$ from where it would have intersected the left parallel (the intersection being designated by another dot or the tip of Segment $b$ or Segment $c$ ). In particular, this means that the two black dots in Configurations 13 and 14 are not placed symmetrically with respect to the left parallel; the lower left dot has a perpendicular distance of $24.6 \mathrm{~mm}$, and the right-hand one, a corresponding distance of $20 \mathrm{~mm}$ from that line.

\section{Method}

The method has been described previously (Weintraub \& Krantz, 1971, Experiment IV). The fixed part of the figure was drawn in India ink on white paper (line thickness, $.25 \mathrm{~mm}$; dot diam, $1.5 \mathrm{~mm})$. The movable black dot $(1.5 \mathrm{~mm})$ was carried by an acetate overlay. The configuration was viewed through a window, shaped like a racetrack (flattened oval), perpendicular to the line of sight, the lines proceeding to the edge of the window. The $S$ adjusted the vertical position of the movable dot to lie on the continuation of the transversal segment (or its substitute when a was absent) by turning a knob projecting from the box enclosing the stimulus. In all cases, the acute angle. $\mathrm{A}$, between transversal and parallels was $24.2 \mathrm{deg}\left(\tan ^{-1} 0.45\right)$ and the separation between parallels (or their remnants) was $20 \mathrm{~mm}$.

The 96 Ss were undergraduates at The University of Michigan. They had normal corrected acuity and no previous experience with experiments on visual illusions. Each $S$ judged the 16 configurations shown in Fig. 3 , plus from 3 to 6 additional Poggendorff variations that had been included to extend other experimental findings (for example, Weintraub \& Krantz, 1971. p. 261, where Experiment IV was expanded). Half of the Ss also made single judgments-whether the right-hand dot, which had been preset by the $F$. appeared too high or too low-in order to compare the group version of the up-and-down method with the adjustment method (see the procedure section of Weintraub \& Krantz, 1971).

\section{Results}

The illusion score is the signed vertical distance between the center of the dot judged to be collinear and the true collinear position. The sign is taken as positive whenever the deviation is in the "Poggendorff" direction as determined from Configuration 1 of Fig. 3 , i.e., a positive score means that the adjusted dot is too low.

For the up-and-down single-judgment trials, our conclusion matches the one reached from Experiment IV (Weintraub \& Krantz, 1971), that, compared to the adjustment method, there were only minor differences in means derived from the up-and-down procedure, but, from an equivalent number of Ss, standard errors of these means were larger. Since we are attempting to organize the material around theoretical issues, only the adjustment data for the 16 stimulus configurations depicted in Fig. 3 will be presented in detail.

Figure 3 gives the mean illusion scores based on one adjustment per configuration for 96 Ss. The standard error of these means is $0.6 \mathrm{~mm}$ (pooled; variance was homogeneous across configurations). The last column of the figure gives results from Experiment III of our previous report, based on group up-and-down estimates. Note that Angle A was smaller there, which partly accounts for the larger illusions. The standard error of the means from Experiment III is about $2.7 \mathrm{~mm}$; so the results are in excellent general agreement. except for 


\begin{tabular}{|c|c|c|c|c|}
\hline \multicolumn{3}{|c|}{ STIMULUS } & \multicolumn{2}{|c|}{ ERROR (MM) } \\
\hline NO. & LINES & FIG. & EXP. VII & EXP. III \\
\hline 1 & abcd & 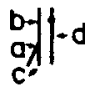 & 11.0 & 22.7 \\
\hline 2 & $a b c-$ & & 9.7 & 14.2 \\
\hline 3 & $a b-d$ & $\int$ & 7.4 & 16.9 \\
\hline 4 & $-b c d$ & & 2.0 & 12.5 \\
\hline 5 & a-cd & & 0.0 & 0.5 \\
\hline 6 & o-c- & 1 & -0.3 & \\
\hline 7 & ab-- & & 5.9 & \\
\hline 8 & $-b c-$ & & 3.2 & \\
\hline 9 & $a--\boldsymbol{d}$ & 1 & 2.0 & 0.8 \\
\hline 10 & $--c d$ & & -1.5 & \\
\hline 11 & $-b-d$ & 11 & 1.5 & \\
\hline 12 & $--d$ & .1 & 1.2 & \\
\hline 13 & $-b--$ & & 3.8 & \\
\hline 14 & $--c-$ & .1 & -3.3 & \\
\hline 15 & $0---$ & $\therefore$ & 1.9 & -0.9 \\
\hline 16 & --- & $\therefore$ & 1.6 & \\
\hline
\end{tabular}

Fig. 3. The 16 modified Poggendorff configurations (Experiment VII) formed by considering all subsets of four basic segments. The four segments are labeled in the picture of Configuration 1, where all four are present. The method-of-adjustment mean illusion measures are shown in the second column from the right (standard error of the mean, based on variance among $96 \mathrm{Ss}$, pooled across conditions, is $0.6 \mathrm{~mm}$ ). Positive values mean that the illusion was in the usual Poggendorff direction (dot set too low). For comparison, results of a previously published experiment (III) are shown in the last column. (The large illusions there are partly accounted for by the use of a smallex acute angle between Segments a and c; pooled standard error was $2.7 \mathrm{~mm}$.)

Configuration 4 (bcd). The group up-and-down results from 48 Ss of Experiment VII are also available and the only sizable discrepancy is for Configuration 1 (abcd), where the up-and-down method yielded an estimated illusion of only $8.67 \mathrm{~mm}$ (pooled standard error =
$1.44 \mathrm{~mm}$ ). This must be sampling error; the difference is of course not significant at the .05 level.

These results indicate that a reliable illusion in the Poggendorff direction is invariably obtained whenever Segment $b$ is present. Segment $c$ produces a reliable increment in the illusion when ab is present ( 1 vs 3,2 vs 7), but otherwise, Segment c produces either little effect (when $b$ is present: compare 13 vs 8,11 vs 4 ) or a sharp decrement, including possibly a negative illusion (when $b$ is absent: compare 15 vs 6,12 vs 10 , and 9 vs 5 ). Segment $d$ also seems to interact with a to increase the illusion when a is present, but to decrease the illusion when $a$ is absent. The addition of Segment a to a configuration produces positive-going shifts in the illusion, enormously enhancing it when $b$ is present, particularly when either $c$ or $\mathrm{d}$ is also present (i.e., $a b$ interacts with $\mathrm{c}$ and $\mathrm{d}$ ).

\section{Discussion}

The above results suggest that the full-fledged Poggendorff illusion (abcd) is produced by a combination of three separate effects on perceived orientation. The least powerful (and least reliable) is the misjudgment of collinearity of three dots alone (Configuration 16); this effect may be accentuated when two of them are replaced by Segment a (Configuration 15). Previous experiments (I and III) produced negative mean illusion scores for Configuration 15, with large standard errors; they are not significantly discrepant from a hypothesis of zero or slightly positive illusion. In this experiment, the standard error is much smaller; moreover, the results from Configurations 15 and 16 are confirmed by Configurations 12 and 9 , where Segment $d$ is added. The size of this effect is around $+1.5 \mathrm{~mm}$, taking all conditions into account.

The second effect is the induction effect of Line Segment b, which is seen in pure form in Configuration 13. If the imagined (transversal) line segment from a dot to the central tip of a vertical segment makes an obtuse angle with that vertical segment, then setting a second dot to lie on the apparent continuation of the imagined transversal produce a Poggendorff-like illusion. In this experiment, the size of this second effect is estimated to be something less than $4 \mathrm{~mm}$; but Experiment IX below, which is devoted to this effect, produced a larger estimate. Configuration 14 suggests that a similar induction situation, involving an acute angle, produces a negative (anti-Poggendorff) effect; Experiments VIII and IX below show that this effect depends very delicately on the stimulus configuration.

The third major effect is that of the actual obtuse angle, $a b$. There are three reasons for treating this as something more than the combination of the first two effects. First, adding a to $b$ produces a marked increase in the illusion, whereas adding a to configurations that 
Table 1

Results of Experiment VII (Millimeters of Illusion)

\begin{tabular}{|c|c|c|c|c|c|c|c|c|}
\hline & \multicolumn{4}{|c|}{ a } & \multicolumn{4}{|c|}{$\bar{a}$} \\
\hline & \multicolumn{2}{|c|}{ b } & \multicolumn{2}{|c|}{$\bar{b}$} & \multicolumn{2}{|c|}{ b } & \multicolumn{2}{|c|}{$\overrightarrow{\mathrm{b}}$} \\
\hline & $c$ & $\bar{c}$ & c & $\vec{c}$ & c & $\overline{\mathbf{c}}$ & $c$ & $\bar{c}$ \\
\hline d & 11.0 & 7.4 & 0.0 & 2.0 & 2.0 & 1.5 & -1.5 & 1.2 \\
\hline $\bar{d}$ & 9.7 & 5.9 & -0.3 & 1.9 & 3.2 & 3.8 & -3.3 & 1.6 \\
\hline & \multicolumn{2}{|c|}{$(+3.7)$} & \multicolumn{2}{|c|}{$(-2.1)$} & \multicolumn{2}{|c|}{ (0) } & \multicolumn{2}{|c|}{$(-3.8)$} \\
\hline
\end{tabular}

do not have Segment $b$ does only a little to accentuate the first effect. Second, the ab configuration is strongly affected by $c$ or $d$ or both, whereas the effects of $c$ and $d$ when $a b$ is not present are nil or even in the opposite direction.

The difficulty in constructing a model of the combined effects of these factors is illustrated by the recasting of the results in Table 1 . (There, absence of a segment is denoted by placing a bar above it, e.g., a $\bar{b} c \bar{d}$ refers to Configuration 6.) For each of the four small matrices, based on a particular combination of a or $\bar{a}$ with $b$ or $\bar{b}$, the effects of $c$ and $d$ are approximately additive. (This arrangement of the data is the only one for which such a result holds, incidentally.) The estimated $c$ vs $\bar{c}$ main effect, shown in parentheses beneath each matrix, varies greatly with the $a b$ combination. If one could take as error-free the estimated $c$ effect with $a b$ present $(3.7 \mathrm{~mm})$ and the estimated $b$ effect in isolation $(3.8 \mathrm{~mm})$, one would be tempted to speculate that the effect of adding $c$ to $a b$ is to restore $b$ as a separate Gestalt, adding its effect to that of the obtuse angle, ab. But, of course, the data are not error-free, and such speculations depend on a much higher degree of precision than what we have so far attained.

\section{EXPERIMENT VIII}

This experiment was designed to provide a more detailed insight into some of the effects uncovered in the previous experiment, including the induction effects of a single segment of the left parallel, b or $c$, represented by Configurations 13 and 14 of Fig. 3.

\section{Stimulus Configurations}

As in Experiment VII, the acute angle formed between transversal (or substitute) and parallels was 24.2 deg. Figure 4 illustrates the configurations; the consecutive numbering of stimuli was continued from the previous experiment. Stimuli 17 and 18, in which the tip of a segment of the right parallel serves to mark the point of apparent collinearity, are adaptations of Stimulus 9 from the previous experiment. Stimuli 19 and 20 are similar adaptations of Stimulus 10.
Configuration 21 matches 12 , except that another parallel has been added midway between the center and right-hand dots. Stimulus 22 matches 6 , except that a line perpendicular to the transversal has been added at the intersection. Stimulus 23 matches 7 , except that a second obtuse angle equal to the first has been added.

Stimuli 24 through 31 represent symmetric dot-location variants of 13 and 14. These eight configurations, shown at the right in Fig. 4, have a double arrow placed next to the movable dot that the $S$ adjusted to apparent collinearity. The transversal was implied by the other dot and the central tip of the line segment. Only four of the eight were actually used in this experiment. The others were ignored in favor of four variants of Stimulus 28 , in which the size of the acute angle and the left-dot distance to the line were investigated.

\section{Method}

The 48 Ss were paid college-age volunteers. Standing Ss looked directly down through a rigid opaque viewing mask at the center of the display, which lay on a table. The mask maintained eye-to-display distance at $54 \mathrm{~cm}$. Narrow eye slits in the mask insured that when the $S$ moved his head out of alignment he could not view the display with both eyes. A simplified apparatus replaced the viewing box and its adjustment knob used in the previous experiment. The stationary portion of the
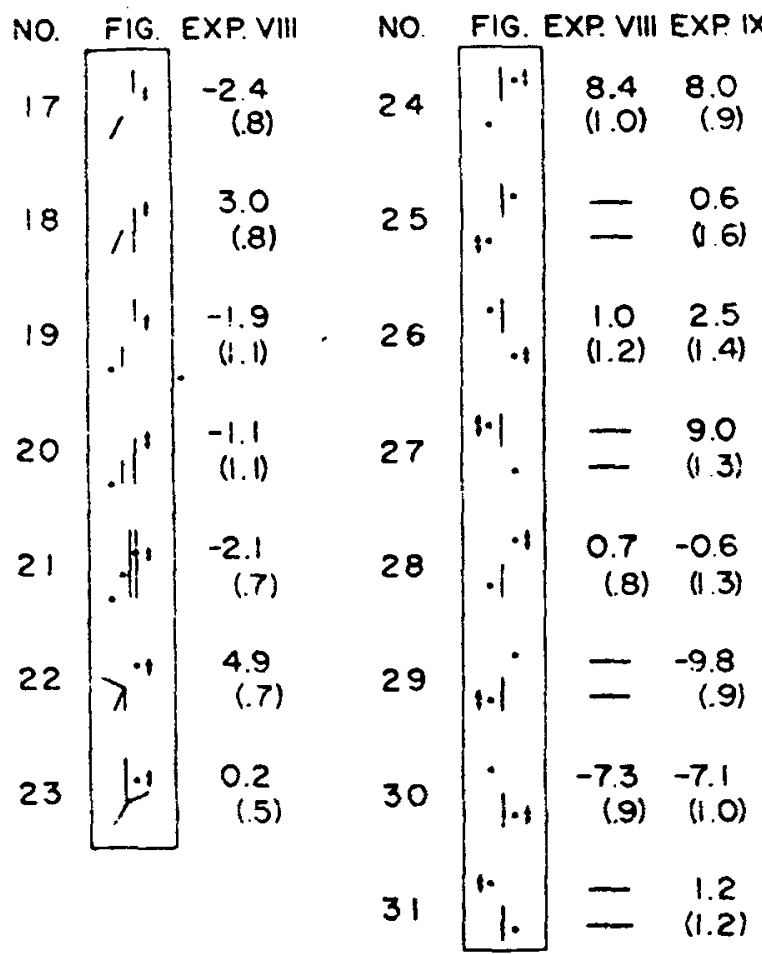

Fig. 4. The 15 configurations of Experiments VIII and IX, together with method-of-adjustment means, with standard errors of the means $(N=48$ and 40 , respectively, in the two experiments) in parentheses. Here, positive values mean that the dot was displaced from veridical toward the bottom of the display (compare Configurations 24 and 30 . both of which produced "Poggendorff"-type effects). 
configuration was drawn in India ink on white $9 \times 12$ in. $(22.9 \times$ $30.5 \mathrm{~cm}$ ) paper, which was recessed flush with the surface of a board. The movable portion of a configuration, a line or dot, was drawn on the underside of an acetate overlay. The left edge of the overlay rested against a raised straightedge so that the movable portion could be adjusted vertically when the $S$ slid the overlay along by hand while keeping the left edge in contact with the straightedge. Each $S$ judged each of the 15 displays once; displays were randomized anew for each $\mathrm{S}$.

\section{Results}

The illusion score was defined as in Experiment VII with positive signs denoting errors toward the bottom of a display. Means and standard errors of the mean are listed in Fig. 4. Data for the four variations of Stimulus 28 where dot location and size of acute angle were manipulated are not shown. None of these stimuli led to a statistically significant amount of illusion, and neither did Stimulus 28.

\section{Discussion}

The initial seven stimulus configurations tended not to resolve any issues but rather to complicate them. Stimuli 17 and 18 showed significant amounts of illusion, with 17 an anti-Poggendorff effect. Compare these outcomes with that of Stimulus 9. Although there is overwhelming evidence that obtuse angles are critical for a Poggendorff effect, Stimulus 18 has no obtuse angle while 17 contains one. The amounts of illusion obtained from Stimuli 19 and 20 are not significant statistically, but they are comparable to the negative illusion obtained from Stimulus 10 . As in 17 vs 18 , the display with the obtuse angle gives a more anti-Poggendorff effect. Stimulus 21 led to a significant negative illusion, even though Stimuli 4 and 12 of the previous experiment produced positive illusions. Stimulus 22 contains no obtuse angle, yet the addition of a right angle (to an acute-angled display, Stimulus 6) led to a moderately strong Poggendorff effect (compare 22 with 2, 6, and 7). It may be concluded from Stimulus 23 that the effects of two obtuse angles cancel (compare with 7). The pristine principle that a Poggendorff effect depends upon an obtuse angle, either present or implied, is questioned by these data.

An unexpected outcome was gleaned from Stimuli 24, 26,28 , and 30 . All prior evidence leads one to expect that left-right mirror images will produce equivalent illusion magnitudes. Therefore, since Stimulus 24 induced $S s$ to set the movable dot $8 \mathrm{~mm}$ too low (a result similar to that of Display iiii in Experiment IV), then the equivalent perceptual conditions in Stimulus 26 also should lead them to set the dot very low. Stimulus 26 produced a nonsignificant Poggendorff illusion. Similarly, if Stimulus 28 produced no illusion, then 30 should act likewise, but such was not the case. The most likely hypothesis seemed astonishing. The nonmovable dot defines an implied transversal, and if the implied subtended angle between the implied transversal and the vertical segment (a primitive parallel) is obtuse, then a Poggendorff effect will emerge. (Stimulus 30 is an "inverted" display and thus an illusion with a negative sign will be obtained.) If the implied subtended angle is acute, then there will be little or no illusion. But such a hypothesis means that the same display will lead to different results, depending upon which dot is movable. As in all previous experiments, only the right dot was movable in Experiment VIII.

Evidence casting doubt upon the hypothesis is that Stimulus 28 was essentially illusion-free, while a sister stimulus, 14, from the previous experiment, gave a significant negative illusion. The only difference between displays was that in Stimulus 28 each dot was located an equal horizontal distance from the vertical line; for 14 , the left dot was further away. (Although none of the four unreported variations of Stimulus 28 gave a statistically significant illusion, it is of interest that close-in dots produced positive illusions and far-out dots produced negative illusions like the difference between .28 and 14.)

\section{EXPERIMENT IX}

All eight possibilities, Configurations 24 through 31 were employed, replicating the even-numbered displays from Experiment VIII. As a further check, Configurations 13 and 14 (only the right dot movable) were replicated from Experiment VII. The 40 Ss were paid college-age volunteers who had not served in previous Poggendorff experiments. The procedures and apparatus were identical to those of Experiment VIII. Each $S$ judged each of the 10 displays once; displays were randomized anew for each $\mathrm{S}$.

\section{Results}

The right column of Fig. 4 presents means and standard errors of the mean. The new data conform closely to the data of Experiment VIII. The replication data for Stimulus 13 (mean $=5.2$, S.E. $=1.2$ ), and Stimulus 14 (mean $=4.9$, S.E. $=1.6)$ correspond well with the outcomes from Experiment VII.

\section{Discussion}

The replication data suggest a modification in the conclusion from Experiment VIII that dot location with respect to the vertical line is a variable that can be ignored. Illusions seem to shift more in the Poggendorff, i.e., positive, direction if the left dot is moved in to a symmetrical position $20 \mathrm{~mm}$ from the vertical line. (Again, compare 13 with 24 and 14 with 28.)

The remarkable and completely consistent finding was that the same configuration provided for either a large or a near-zero Poggendorff effect, depending on which dot was movable. Compare 24 with 25,26 with 27 , etc. Left-right mirror-image differences were small (cf. 24 
with 27,25 with 26,28 with 31,29 with 30 ). If the direction and amount of illusion is defined with respect to the configuration rather than with respect to the bottom of the O's visual field, then pairs of displays with up-down symmetry gave equivalent illusions (cf. 24 with 30,25 with 31,26 with 28,27 with 29). Up-down asymmetry was expected on the basis of Experiment $\mathrm{V}$ (Weintraub \& Krantz, 1971) and data gathered by Weintraub and Virsu (1972), but it did not materialize.

Thus, only an "obtuse-angled" display led to an illusion. However, each display contains both an acute and an obtuse implied angle, and so the choice of fixed dot defining an implied transversal was critical. Consider that, for a configuration like 28 , the movable dot was set close to true collinearity when apparent collinearity was achieved. If one looks at (scans? attends to?) the three collinear elements in reverse order, then they no longer appear collinear. (An $8-\mathrm{mm}$ adjustment of the end dot was necessary, as in Stimulus 29.) The result shows very clearly that Poggendorff-like displays are viewed asymmetrically, and that perceived orientation refers to directed line segments.

\section{REFERENCES}

Weintraub, D. J., \& Krantz, D. H. The Poggendorff illusion: Amputations, rotations, and other perturbations. Perception \& Psychophysics, 1971, 10, 257-264.

Weintraub, D. J., \& Virsu, V. Estimating the vertex of converging lines. Angle misperception? Perception \& Psychophysics, $1972,11,277-283$.

(Received for publication January 8, 1973; revision received July 6,1973 .) 